\title{
Model Integration Workflow for Keeping Models up to Date in a Research Simulator
}

\author{
Torsten Gerlach, Umut Durak and Jürgen Gotschlich \\ Institute of Flight Systems, German Aerospace Center (DLR), Lilienthalplatz 7, Braunschweig, Germany \\ \{torsten.gerlach, umut.durak, juergen.gotschlich\}@dlr.de
}

Keywords: Model Integration, Flight Simulators, Model based Design and Development, Simulink Coder.

Abstract: $\quad$ Flight simulators can be categorised as research simulators, engineering simulators and training simulators. Research simulators can be introduced as both test beds for flight simulator research and computational tools for flight systems and human factors research. While engineering simulators are utilised for systems development, training simulators are used for flight training. The models that are used in training simulators and also in engineering simulators are more mature and stable. On the other hand, the models in research simulators are subject to a constant change. While Model Based Design and Software Development has brought us agile model development workflows, so that modellers can update their models more easily, it came up with some serious systems integration and testing problems, so systems developers need to establish mechanisms to tackle frequent behaviour and interface changes. DLR's Institute of Flight Systems (FT) has a long tradition in flight research and simulation of various flight vehicles. Currently a modern research simulator facility is being operated at DLR Braunschweig -AVES (Air Vehicle Simulator). AVES is designed such that interchangeable cockpits of rotorcraft (EC135) and airplanes (A320) can be operated on motion and fixed-base platforms according to the particular needs. 2Simulate is the enabling real-time simulation infrastructure of the AVES. This paper presents 2Simulate model integration workflow based on Mathwork's Simulink Coder.

\section{INTRODUCTION}

Till late 1920s, when Edward Link built one of the early examples of flight simulators, they have been important elements of aviation. These first examples which were known as Blue Box, were designed to train pilots for instrumented flight (Allerton, 2009). Before digital era, flight simulators became well accepted as training aids by many aircraft operators. Then as the fidelity of flight simulators increased, engineering standards to build flight simulators for flight training were developed.

As flight simulators became de facto tools in flight training, they were also started to be used in aircraft development. After 1980s, testing and validation of aircraft systems started to be done in engineering flight simulators. Thus, potentially dangerous and expensive flight tests could be avoided (Allerton, 1999).

In 80 s, aeronautics research community was also using flight simulators for developing and experimenting advanced concepts. ATTAS Simulator from German Aerospace Center (DLR)
(Saager, 1990) (Klaes, 2000), NASA Crew Vehicle Systems Research Facility in Ames Research Center (Sullivan \& Soukup, 1996) and Visual Motion Simulation and Cockpit Motion Facility from Langley Research Center (Smith, 2000) were some of the first examples of research flight simulators. Some of the recent ones are Air Vehicle Simulator (AVES) of German Aerospace Center (DLR) (Duda et al., 2013), HELIFLIGHT from the University of Liverpool (White \& Padfield, 2006), NASA Ames Vertical Motion Simulator (Advani et al., 2002) and SIMONA of the Delft University of Technology (Stroosma et al., 2003).

The organisation of a flight simulator is structured around the flight dynamics model. There may be various components that supports or works with flight dynamics model, like aerodynamics model, landing gears model, weather model, engine model and subsystem models. The architecture of these models varies from simulator to simulator. They can either be implemented as a single model or various models interacting in a tightly coupled manner. The other important components like 
SIMULTECH 2014 - 4th International Conference on Simulation and Modeling Methodologies, Technologies and Applications

control loading, instructor station, motion system, visual system, instrument displays either provide inputs to these models or present their results to the pilot as cues (Allerton, 2009).

Research simulators have been used as the test beds for flight simulator, flight systems and human factors research. So, while the models that are used in training simulators and even in engineering simulators are more mature and stable, the models in research simulators are subject to a constant change.

Recent advances in Model Based Design and Software Development (MBDSD) have brought aeronautics community agile model development workflows. So that model development is integrated to product development employing mature code generation practices (Ruff et al., 2012). Models developed to design the products now became the bases for code generation to be deployed in the product.

For research flight simulators with MBDSD, the models that are built to study overall systems (e.g. Flight Dynamics Model) and subsystem (e.g. Flight Warning Computer Model) behaviour became the bases for generating code to be deployed in real time flight simulators. These models serve for researchers that exercise various aspects of aircraft in their desktop environments, and for simulator developers to simulate aircraft system and sub systems.

Research simulator developers need to establish mechanisms to tackle frequent behaviour and interface changes in models. And constant model changes in a research habitat can only be enabled with a model integration workflow in the systems development. But flight simulator literature lacks in reporting any efforts.

There are some recommended practices from the aerospace industry for model based flight systems design and development. Estrada et al. introduce best practices for developing DO-178 compliant software using Model-Based Design and Development (Estrada, R.G. et al., 2013). Miller presents automatic flight code generation practices in Northrop Grumman (Miller, 2007) and introduces a use case from desktop simulation to Hardware in the Loop testing. BAE Systems has a model based flight control systems development process (Fielding, 2010). Fielding presents a process starting from aerodynamic dataset generation to flight clearance of the aircraft. In this process he mentions the use of engineering simulators for model based flight control system design. Nixon states that in F-35 project MBDSD forced them to reinterpret traditional software development process for flight control systems (Nixon, 2004). He introduces Lockheed Martin Aeronautics practices of MBDSD.

On the other hand, there exists a vast amount of effort to develop integration workflows for their model based developed software component. In one of them (Guido and Thompson, 2008) from Mathworks, authors propose a workflow to develop software components to be integrated to Automotive Open System Architecture (AUTOSAR). AUTOSAR specifies the architecture to integrate functional applications over a hardware abstracting runtime environment in automotive electronic control units. The presented workflow enables modellers to develop an infrastructure compliant model development and seamless integration over a standard architecture.

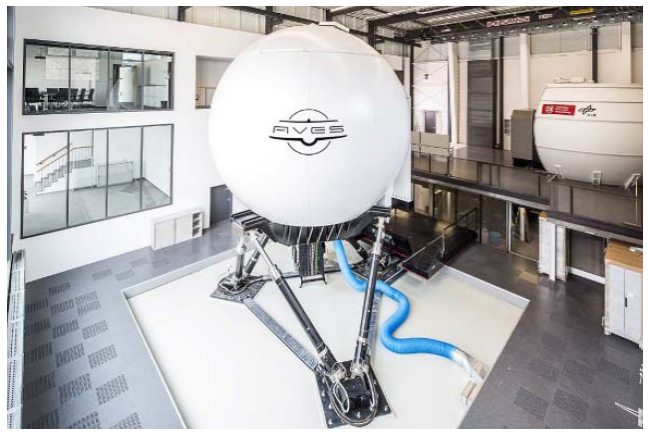

Figure 1: DLR AVES.

DLR's Institute of Flight Systems (FT) has a long tradition in flight research and simulation of various flight vehicles. Currently AVES, a modern research simulator facility is being operated at DLR Braunschweig. AVES is designed such that interchangeable cockpits of rotorcraft (EC135) and airplanes (A320) can be operated on motion and fixed-base platforms according to the particular needs. 2Simulate is the enabling real-time simulation infrastructure of AVES. All simulator software components are integrated over this infrastructure. This effort adopts best practices from both aerospace and automotive industries. It tackles the model integration problem of research flight simulators by developing a model integration workflow for the indigenous simulator infrastructure, namely 2Simulate. The motivation is to contribute to flight simulator development by introducing a model integration workflow for institutionalizing MBDSD.

The paper presents the Mathwork's Simulink Coder based model integration workflow of 2Simulate infrastructure in AVES facility. This workflow provides the users of AVES a shortened time to simulator after they updated their models. 
First the reader will be introduced to 2Simulate. Following the presentation of proposed model integration workflow, how the 2Simulate Model Control is designed to enable this workflow will be discussed. Sample model integration will then be provided to exemplify the concepts and technologies introduced.

\section{2SIMULATE}

AVES was developed based upon the strategy to employ reusable, flexible, standardized and properly validated software modules. 2Simulate is an overall simulation framework to facilitate integrating a wide range of models and simulation components like external devices, data recorders or image generators. (Gotschlich et al., 2014).

It is a $\mathrm{C}++$ real-time distributed simulation framework which is composed of three components, namely 2Simulate Real-Time Framework (2SimRT), 2Simulate Model Control (2SimMC) and 2Simulate Control Center (2SimCC) (Figure 2).

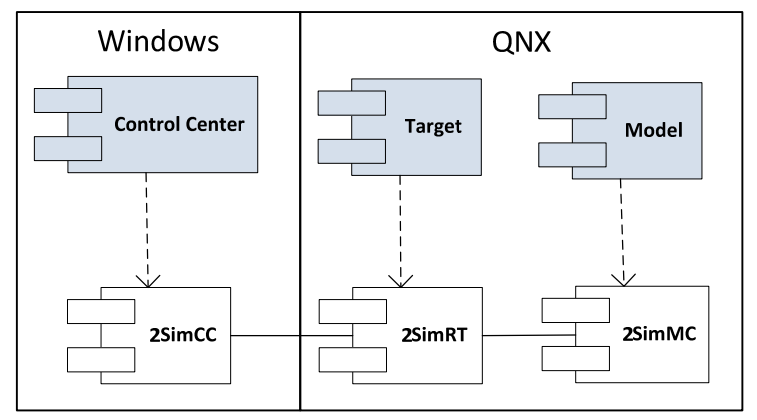

Figure 2: 2Simulate Components.

2SimRT is the core simulation framework of 2Simulate that provides deterministic scheduling and controlling of real-time tasks. It comes as libraries and API header files for Windows to support soft real time implementations like desktop simulators, or QNX to support hard real time implementations like full flight simulators. Hard real time use case is targeted in the scope of this paper. Any simulation application that is based on 2SimRT is called a Target. Targets possess various real-time tasks that are implemented utilizing the 2SimRT API. TSimModel is one of these real-time tasks used for integrating Simulink models. Tasks can be programmed using their pre- and post-initialization and pre- and post-process callbacks. 2SimRT also provides a Common Database to manage the data flow through the internal and external interfaces (Figure 3).

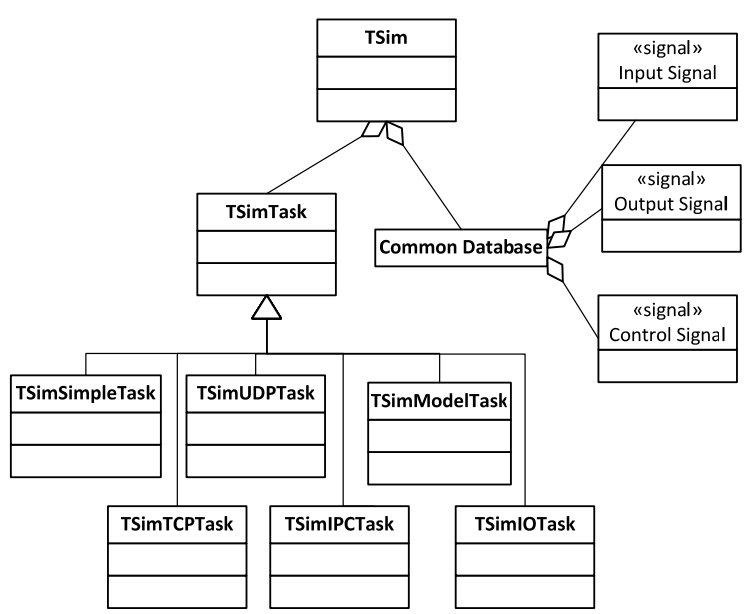

Figure 3: Main 2Simulate Classe.s

2SimMC is the enabler of model integration workflow. It is composed of 2 Simulate Model Control Source (2SimMC-Source) that abstracts model interfaces for 2SimRT, and 2Simulate Model Control Scripts (2SimMC-Scripts) that includes Simulink Coder Target Language Compiler files (TLC files) to specify the 2Simulate target and mfiles to conduct the code generation and build process.

$2 \mathrm{SimCC}$ is the graphical user interface that is configured to a Control Center for specific needs. It is a Windows executable which can be customized via configuration files called 2 SimCC project files. Control Center can run, pause or stop various Targets. Besides, it accesses the Target Data Dictionaries which can be defined as the data access mechanisms and enables presenting or editing Target data at runtime. It can also enable user management to define and enforce user access rights.

\section{INTEGRATION WORKFLOW}

Model integration workflow is triggered if any of the simulator models in the simulator are updated. As soon as the update is tested and verified in the modeling environment, which is Matlab/Simulink, the modeler would like to deploy it to its target. The process assumes that the modeler assures that the model is valid and correct.

Proposed workflow starts with a static model checking step before continuing to generating source code. Checking a model for modeling guidelines will provide a set of valuable information about what best practices or guidelines are violated. Thus it contributes to the quality of the model (Fey and 
Stürmer, 2007). Measuring and assessing the quality of the model for code generation has various aspects including structured and automated testing, coverage analysis, complexity analysis, modeling guidelines (Stürmer and Pohlheim, 2012). Matlab Model Advisor is employed as a starting point in this step to check the mode for conditions and configurations that may lead to generation of inaccurate and inefficient code (The MathWorks, Inc., 2007) .

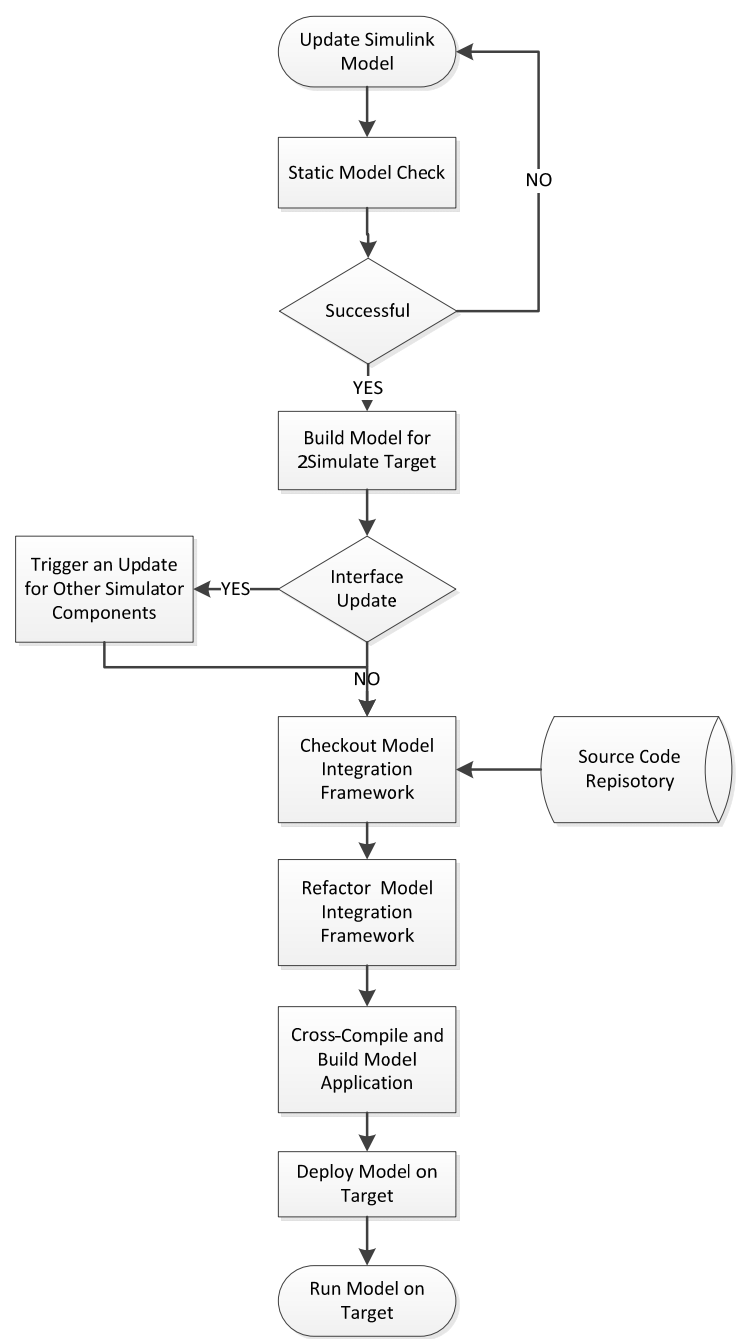

Figure 4: Model Integration Workflow.

As depicted in Figure 4, the next step is building the model for 2Simulate target. The Simulink model can be used with 2Simulate after it has been converted into $\mathrm{C}++$ code using Mathworks Simulink Coder (The Mathworks, Inc., 2014a). A part of the Simulink Coder is the Target Language Compiler. It specifies the code generation (The Mathworks, Inc., 2014b) utilizing so called system target files, which can be customized for specific needs. 2Simulate has such a set of customized system target files. They embed 2SimMC into the model code during the code generation. Thus, an auto-generated model is readily available for 2 Simulate model task. At this step, these set of target files are employed. The details of 2Simulate system target files will be presented in the next section.

The changes in the model interface are traceable over the signal specifications for the model generated, while code generation process. It is almost clear that any change in the model interface will cause an update in the other simulator components that depend on these signals. So the next step of the integration workflow is to trigger an update process for the other components if any change in the model interface is identified.

The next two steps in the process aims at preparing the model application source project. At first Model Integration Framework is checked out from the source repository. This framework is a wrapper for the generated model code. It creates a 2SimRT target using the generated model code. This wrapper code is refactored automatically for model specific parameters. As an example, the solver step size of the model is set as the frequency of the model task in the Model Integration Framework code. After refactoring model application code is ready for compilation.

The rest of the process is to cross-compile the application code for the QNX target and deploy the generated image to the target system. At the end of the workflow, the updated model becomes readily runnable at the target system.

\section{2SIMULATE MODEL CONTROL}

In this section, components of 2SimMC, 2SimMCScripts and 2SimMC-Source will be introduced.

There are two types of scripts in 2SimMCScripts. A Matlab script TSimModelBuilder.m is in charge for Matlab automation for every step that is depicted in Figure 4. And TLC files are used to specify the 2Simulate target.

TSimModelBuilder.m makes use of Matlab command line utilities for controlling Model Advisor, Simulink Coder and calling some external executables for source control, cross-compilation and secure shell. It also conducts refactoring in the Model Integration Framework code employing file and string manipulation utilities of Matlab. Below is a representative code extract from TSimModelBuilder.m 


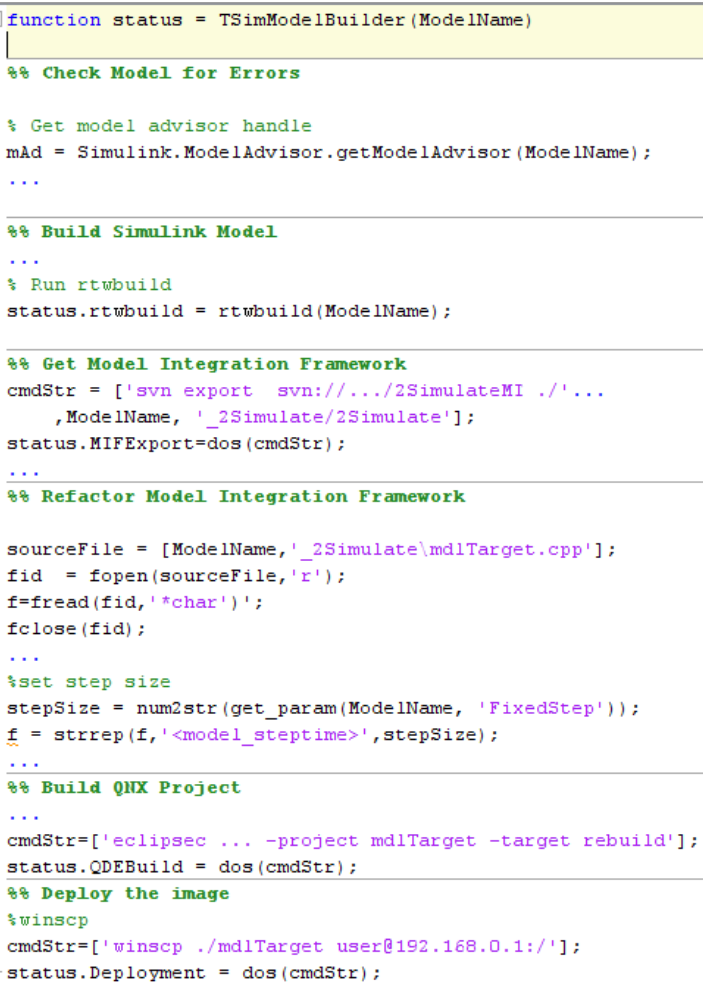

Simulink Coder allows its users to select a target for code generation. Target Language Compiler on the other hand, provides capabilities to specify targets through customizing the generated code to produce platform or application specific code. It transforms model.rtw, the intermediate form of Simulink block diagram into $\mathrm{C}$ or $\mathrm{C}++$ code. Code generation is controlled by TLC files. TLC files have uses a syntax like Perl or other scripting languages, augmented with data handling capabilities of Matlab (The Mathworks, Inc., 2014b). One can create and modify the generated code, generation time data processing with TLC directives and accessing model structure captured in model.rtw. It provides looping, file $\mathrm{I} / \mathrm{O}$, scoping type powerful scripting tools.

For 2Simulate a target specification called grt 2Simulate is implemented by 2SimMC-Scripts TLC files. These files extend generic real-time target provided by Simulink Coder. The top level entry point is grt_2Simulate.grt. As presented in Figure 5, it first calls codegenentry.tlc to generate model code and then calls all eight 2Simulate TLC files to generate 2SimMC-Component code.

2SimMC-Component code is composed of sources for a 2SimRT task, model, data dictionary, model defines and specifications for input and output signals. Task and model TLC files extend

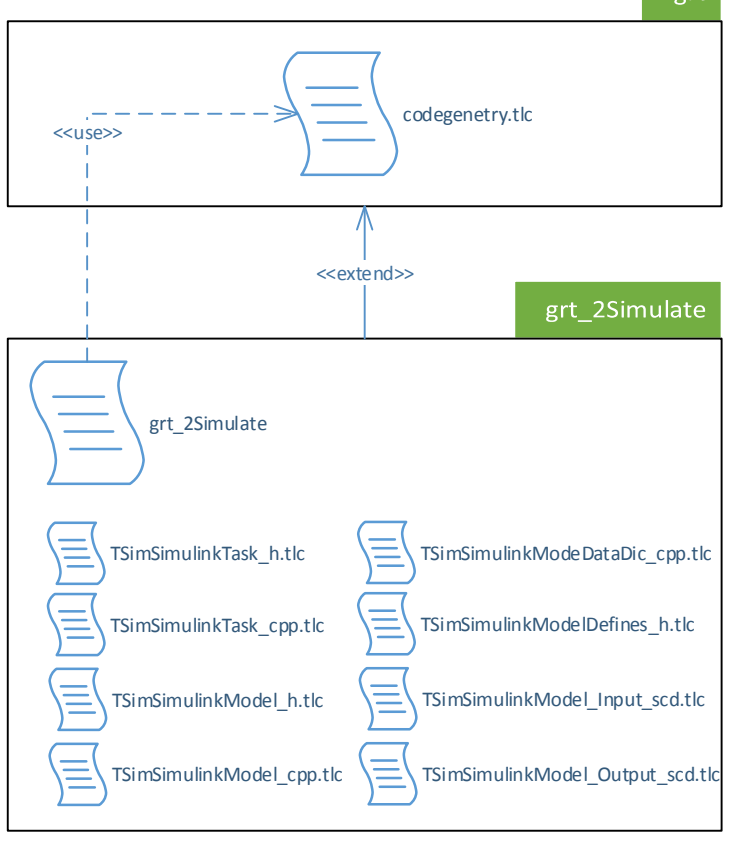

Figure 5: 2Simulate System Target File Structure.

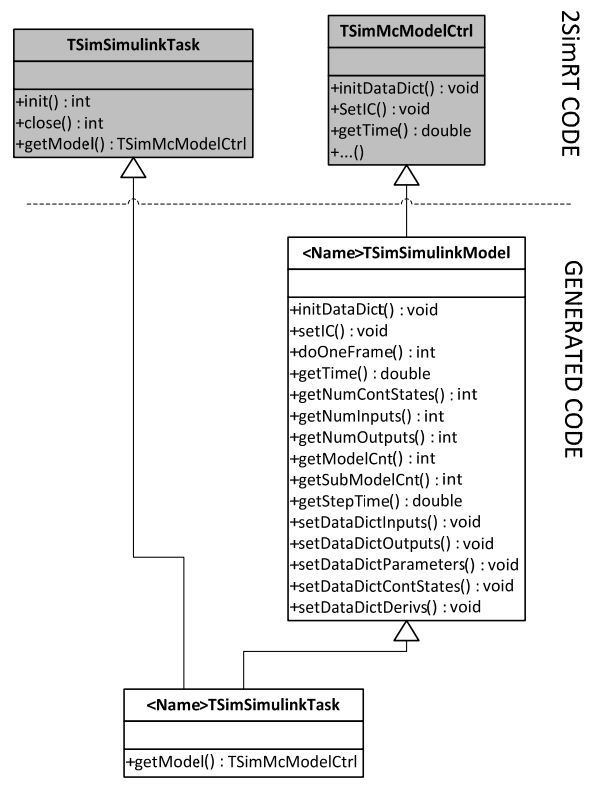

Figure 6: 2SimMC-Component Classes.

2SimRT API and glue it with generated model code (Figure 6). $\quad<$ Name $>$ TSimSimulinkModel class inherits from TSimMcModelCtrl from 2SimRT API and includes $<$ name $>$.h that enables it to access Simulink model code. On the other hand $<$ Name $>$ TSimSimulinkTask class inherits from both model class and TSimSimulinkTask from 2SimRT 
API, so that one can use this class to create a schedulable 2SimRT task for the Simulink model.

TSimSimulinkModelDataDict_cpp.tlc generates a source file for setters and getter of the data dictionary. These setters and getters allow their users to access and modify model parameters, contentious states and state derivatives as well as model input and outputs.

$<$ name $>$ ModelDefines. $h$ is a helper file to specify model wide global parameters. Below is an excerpt from TSimSimulinkModeDefines_h.tlc that demonstrates a sample scripting in a TLC fille.

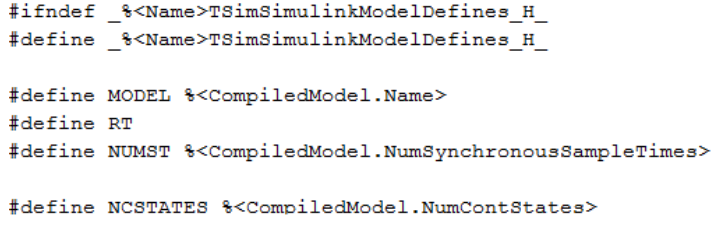

Above one can see how TLC tokens are used to get information from the model and incorporate them in the source code. As an example, number of continuous states are defined using the token CompiledModel.NumContStates.

As a final step, Target Language Compiler is used as a model-to-text transformation tool for generation signal specifications for inputs and outputs of the model as ASCII files. With these two TLC files, TSimSimulinkModel Input_scd.tlc and TSimSimulinkModel_Output_scd.tlc, the limits of Target Language Compiler are pushed to generate project or platform specific files. Below is an excerpt from these TLC files.

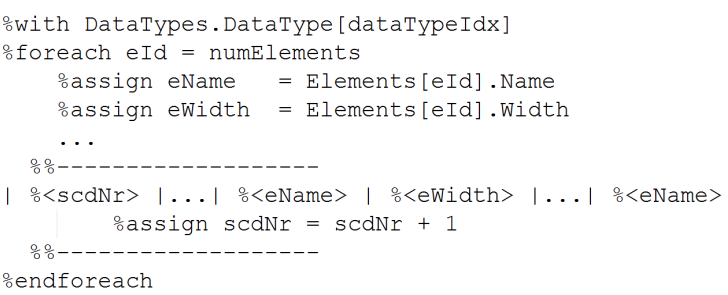

\section{A SAMPLE MODEL INTEGRATION}

In this section, the model integration workflow that has been introduced will be used for a sample Simulink model. We will use an open source Quadrotor Flight Dynamics and Control Model (Mathworks File Exchange, 2013) (Figure 7) which implements flight dynamics and control algorithms from Bouabdallah's work (Samir, 2007).

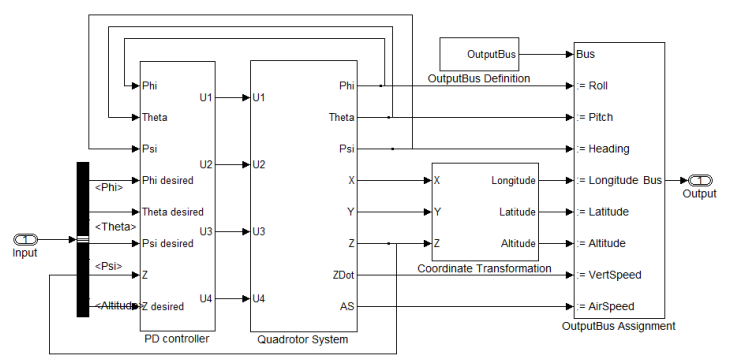

Figure 7: Simulink Model of Quadrotor Flight Dynamics and Control.

As we run TSimModelBuilder.m for quadrotor.mdl, the process will lead us through the steps of Figure 4 till to the deployment of the binaries for the model application to the specified target.

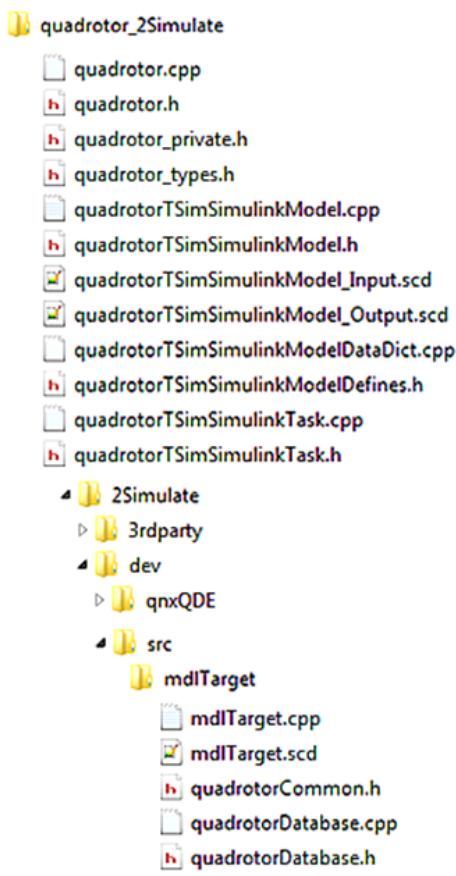

Figure 8: Generated Files.

The workflow leads to a structure that is presented in Figure 8. The files generated by the Target Language Compiler stay under the root of quadrotor_2Simulate directory. The Model Integration Framework that includes 3rdparty dependencies, model application source code and QNX Development Environment project is checked out to 2Simulate directory.

The main routine of the model application code can be found in mdlTarget.cpp. As given in the following code excerpt, 2SimRT application 
possesses three tasks. The first one is for the model, the second one is for the Control Center and the last one is for UDP communication to other simulation components.

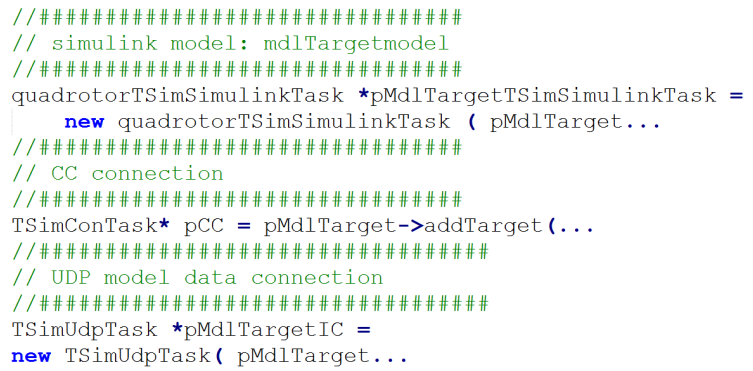

Then the generated source files are crosscompiled to QNX and the image is deployed to a QNX target using the open source tool WinSCP.

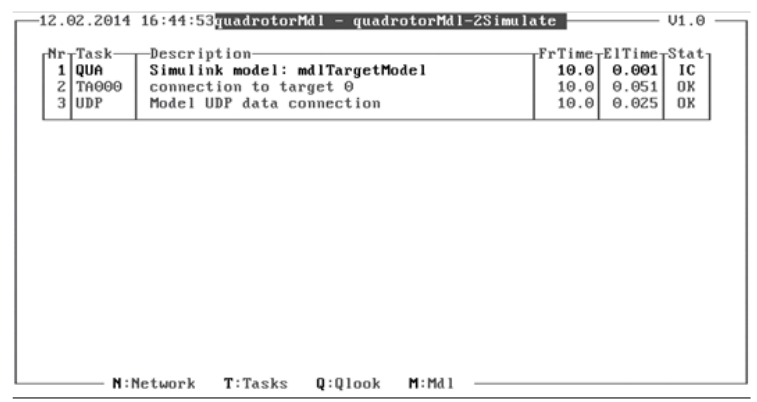

Figure 9: Console Running quadrotorMdl.

The Model Integration Workflow presented in this paper ends when the image of the model application is deployed to the specified target. The deployment scenarios launch mechanisms and network settings vary between simulators. Figure 9 presents a snapshot from a QNX console that runs the deployed model application image.

\section{CONCLUSIONS}

As in other research flight simulators, model update is a constant process also in DLR AVES. Flight systems researchers work for extending and enhancing their models or their systems. The presented Model Integration Workflow intends to make shortened Time-to-Deployment. Furthermore, with automated code generation and deployment process, man made errors are avoided.

With this workflow, the flight systems researchers, that use MBDSD practices, are supported for easy and fast integration and deployment of their models. Users of this workflow can integrate and deploy their models in AVES within a minimum time.

This workflow is currently operated over the Matlab command prompt. While it supports update to deployment process, it lacks in intuitive user interface for configuration and execution. Furthermore it has no support for run time monitoring and debug. Future plans include developing a graphical user interface and Simulink blocks for run time monitoring. Thus the users of the workflow will be able to monitor and debug their models that run in AVES from Simulink.

\section{REFERENCES}

Advani, S., Giovannetti, D. \& Blum, M., 2002. Design of a Hexapod Motion Cueing System fir NASA Ames Vertical Motion Simulator. In AIAA Modeling and Simulation Technologies Conference and Exhibit. Monterey, California, 2002. AIAA.

Allerton, D.J., 1999. The Design of a Real-Time Engineering Flight Simulator for the Rapid Prototyping of Avionics Systems and Flight Control Systems. Transactions of the Institute of Measurement and Control, pp.51-62.

Allerton, D., 2009. Principles of Flight Simulation. West Sussex, United Kingdom: John Wiley \& Sons, Ltd.

Duda, H., Gerlach, T., Advani, S. \& Potter, M., 2013. Design of the DLR AVES Research Flight Simulator. In AIAA Modeling and Simulation Technologies (MS) Conference. Boston, MA, 2013. AIAA.

Estrada, R.G., Sasaki, G. \& Dillaber, E., 2013. Best practices for developing DO-178 compliant software using Model-Based Design. In AIAA Infotech@Aerospace (I@A) Conference. Boston, MA, 2013. AIAA.

Fey, I. \& Stürmer, I., 2007. Quality Assurance Methods for Model-based Development: A Survey and Assessment. In SAE World Congress \& Exhibition. Detroit, Michigan, 2007. SAE.

Fielding, C., 2010. Model-Based Design on Flight Control Systems. In Mathworks Model-Based Design Conference. Daventry, UK, 2010. Mathworks, Inc.

Gotschlich, J., Gerlach, T. \& Durak, U., 2014. 2Simulate: A Distributed Real-Time Simulation Framework. In ASIM STS/GMMS Workshop 2014. Reutlingen, Germany, 2014. ASIM.

Guido, S. \& Thompson, R., 2008. Development of AUTOSAR Software Components within ModelBased Design. In Proc. SAE World Congress \& Exhibition. Detroit, MI, 2008. SAE.

Klaes, S., 2000. ATTAS Ground Based System Simulator -An Update-. In AIAA Modeling and Simulation Technologies Conference and Exhibit. Denver, CO, 2000. AIAA. 
SIMULTECH 2014 - 4th International Conference on Simulation and Modeling Methodologies, Technologies and Applications

Mathworks File Exchange, 2013. PD Control Quadrotor. [Online] Available at: http://www.mathworks.com/matlabcentral/fileexchang e/41149-pd-control-quadrotor-simulink [Accessed 10 February 2014].

Miller, R., 2007. Automatic Code Generation at Nortrop Grumman. In Mathworks Aerospace and Defence Conference. Manhattan beach, CA, 2007. Mathworks, Inc.

Nixon, D.W., 2004. Flight Control Law Development for the F-35 Jointr Strike Fighter. In The Mathworks International Aerospace and Defence Conference. Newton MA, 2004. Mathworks, Inc.

Ruff, R., Stephans, C. \& Mahapatra, S., 2012. Applying Model-Based Design to Large-Scale Systems Development: Modeling, Simulation, Test, \& Deployment of a Multirotor Vehicle. In AIAA Modeling and Simulation Technologies Conference. Minneapolis, Minnesota, 2012. AIAA.

Saager, P., 1990. Real-Time Hardware-in-the-Loop Simulation for 'ATTAS' and 'ATTHeS' Advanced Technology Flight Test Vehicles. In AGARD Guidance and Control Panel, 50th Symposium. Izmir, Turkey, 1990. NATO.

Samir, B., 2007. Design and Control of Quadrotors with Application to Autonomous Flying. Ph.D. Thesis. Lausanne: École Polytechnique Fédérale de Lausanne.

Smith, R.M., 2000. A Description of the Cockpit Motion Facility and the Research Flight Deck Simulator. In AIAA Modeling and Simulation Technologies Conference and Exhibit. Denver, CO, 2000. AIAA.

Stroosma, O., van Paassen, R. \& Mulder, M., 2003. Using the Simona Research Simulator for Human-Machine Interaction Research. Austin, Texas, 2003. AIAA.

Stürmer, I. \& Pohlheim, H., 2012. Model Quality Assessment in Practice: How to Measure and Assess the Quality of Software Models During the Embedded Software Development Process. In Int. Congress of Embedded Real Time Software and Systems (ERTS 2012). Toulouse, France, 2012. ERTS.

Sullivan, B.T. \& Soukup, P.A., 1996. The NASA 747-400 Flight Simulator: A Natonal Reseource fir Aviation Safety Research. In AIAA Flight Simulation Technologies Conference. San Diego, CA, 1996. AIAA.

The MathWorks, Inc., 2007. Matlab Product Help: Consulting Model Advisor. Help Document. Natick, MA: The MathWorks, Inc. The MathWorks, Inc.

The Mathworks, Inc., 2014a. Simulink Coder: Generate C and $C++$ Code frim Simulink and Stateflow. [Online] Available

at: http://www.mathworks.com/products/datasheets/pdf/si mulink-coder.pdf [Accessed 08 April 2014].

The Mathworks, Inc., 2014b. Simulink ${ }^{\circledR}$ Coder $^{\mathrm{TM}}$ Target Language Compiler. Help Document. Natick, MA: The MathWorks, Inc.

White, M.D. \& Padfield, G.D., 2006. The Use of Flight Simulation for Research and Teaching in Acedemia. In AIAA Atmospheric Flight Mechanics Conference and Exhibit. Keystone, CO, 2006. AIAA. 